\title{
Model eyes with curved multilayer structure for the axial resolution evaluation of an ophthalmic optical coherence tomography device
}

\author{
Zhenggang $\mathrm{Cao}^{*, \S}$, Zengqian Ding ${ }^{\dagger+}$, Zhixiong $\mathrm{Hu}^{* \boldsymbol{9}, * *}$, Wen Qiao ${ }^{\dagger+*}$, Wenli Liu* \\ and Xiaojun Chen ${ }^{\S, \|, * *}$ \\ *National Institute of Metrology, Division of Medical and \\ Biological Measurements, Beijing 100029, P. R. China \\ $\dagger$ Optoelectronics and Energy $\&$ Collaborative Innovation \\ Center of Suzhou Nano Science and \\ Technology College of Physics, \\ Soochow University, Suzhou 215006, P. R. China \\ *Key Laboratory of Advanced Optical Manufacturing \\ Technologies of Jiangsu Province and Key Laboratory of \\ Modern Optical Technologies of Education \\ Ministry of China, Soochow University \\ Suzhou 215006, P. R. China \\ ${ }^{\S}$ School of Mechanical Engineering, \\ Shanghai Jiao Tong University, Shanghai 200240, P. R. China \\ Thuzhixiong@nim.ac.cn \\ "xiaojunchen@sjtu.edu.cn
}

Received 25 October 2017

Accepted 8 January 2018

Published 8 February 2018

\begin{abstract}
Optical coherence tomography (OCT) has been widely applied to the diagnosis of eye diseases during the past two decades. However, valid evaluation methods are still not available for the clinical OCT devices. In order to assess the axial resolution of the OCT system, standard model eyes with micro-scale multilayer structure have been designed and manufactured in this study. Mimicking a natural human eye, proper Titanium dioxide $\left(\mathrm{TiO}_{2}\right)$ materials of particles with different concentrations were selected by testing the scattering coefficient of PDMS phantoms. The artificial retinas with multilayer films were fabricated with the thicknesses from 9.5 to 30 micrometers using spin coating technology. Subsequently, standard OCT model eyes were accomplished by embedding the retina phantoms into the artificial frames of eyes. For ease of measurement processing, a series of model eyes were prepared, and each contained films with three kinds of thicknesses. Considering the traceability and accuracy of the key parameters of the
\end{abstract}

${ }^{* *}$ Corresponding authors.

This is an Open Access article published by World Scientific Publishing Company. It is distributed under the terms of the Creative Commons Attribution 4.0 (CC-BY) License. Further distribution of this work is permitted, provided the original work is properly cited. 
standard model eyes, the thicknesses of multilayer structures were verified using Thickness Monitoring System. Through the experiment with three different OCT devices, it demonstrated the model eyes fabricated in this study can provide an effective evaluation method for the axial resolution of an ophthalmic OCT device.

Keywords: Optical coherence tomography, metrology, optical standards and testing, spin coating.

\section{Introduction}

Optical coherence tomography (OCT) is a threedimensional (3D) imaging technology with features of nonintrusion and high resolution. Since the OCT concept was proposed by Fujimoto research group in $1991,{ }^{1}$ great progress has been made in this technology, especially in the field of ophthalmology. Although ophthalmic OCT technology had received permission for the clinical diagnosis in America, Europe and Asia, its first international standard was not released until April 2015. ${ }^{2}$ Specific standard phantoms as well as simple evaluation methods are required for not only manufacturers and clinical users, but also the third-party test institutions, aiming at registration tests, regular quality control and product comparison. ${ }^{3}$

Compared with traditional medical imaging technologies such as Computed Tomography (CT), Magnetic Resonance Imaging (MRI) and ultrasound imaging, OCT can precisely provide crosssectional information of target tissues with high axial resolution. As is well known, axial resolution is one of the most critical parameters for the OCT image quality, which determines the diagnosis accuracy. Currently, there are two common methods, i.e., Point Spread Function ${ }^{4-6}$ (PSF) and the use of a phantom, for the evaluation of the axial resolution of an OCT device. Since post-processing of PSF is very complicated and not suitable for clinical applications $^{7}$, the use of a phantom plays a more important role in clinical standardization and validation due to the advantages of its intuitiveness and efficiency.

Recently, several research groups have been working on manufacturing suitable phantoms for the evaluation of ophthalmic OCT devices. Agrawal et al. and Rowe et al., focused on how to manufacture model eyes mimicking the shape of natural eyes and the scale of real retina ${ }^{8-10}$, which however, was not related to the evaluation method for measuring the axial resolution of an OCT device. Using a novel processing method, $\mathrm{Gu}$ et al. fabricated a phantom suitable for standardized measurements of the axial resolution of a research-grade OCT device, ${ }^{11}$ in which the adjustment process was too complicated for clinical applications. Curatolo et al. manufactured standard phantoms with 3D features using Replica-Molding method to detect the parameters of OCT devices, ${ }^{12}$ however, the materials of these phantoms are limited. Tomlins et al. ${ }^{13}$ designed and fabricated a phantom with the method of Femtosecond Optical Pulses, but high cost and critical requirements for this technology are major problems for clinical applications. Agrawal et al. fabricated a practicable phantom, however, it was composed of the layers with same thickness leading to low efficiency, and the dissimilarity of physical characteristics compared with natural eyes may not guarantee the measurement accuracy. ${ }^{7} \mathrm{Hu}$ et al. proposed a method of manufacturing a 3D test target with 3D printing technology for measuring the axial resolution of an ophthalmic OCT device, nevertheless, the highest axial precision of the $3 \mathrm{D}$ printers is $16 \mu \mathrm{m}$, which is still too low to detect that of OCT devices. ${ }^{13}$

In this study, novel standard model eyes were designed and fabricated, and the evaluation criterion for the axial resolution of an ophthalmic OCT device was proposed. The advantages of the model eyes include: (1) the artificial arc retinas that mimic human ones; (2) the layers with three kinds of thicknesses in the artificial retina that guarantee efficiency; (3) the various layers with different scattering coefficient PDMS-Titanium dioxide $\left(\mathrm{TiO}_{2}\right)$ materials that avoid the inhomogeneity of signals. Finally, the model eyes were used to detect the axial resolution of three OCT systems, including one clinical and two research-grade devices. The results indicated that it provided an effective method to rapidly evaluate the axial resolution of an ophthalmic OCT device.

\section{Material Selection}

Commonly, light propagating in biological tissues could be scattered and absorbed by different 
compositions such as cells, fiber, hemoglobin, melanin, water, and so on, ${ }^{15}$ By collecting and analyzing the scattering and absorbing light properties, the OCT system can obtain a cross-sectional image of the target tissue. In order to mimic a real tissue, the materials of retina phantoms should have similar optical properties with real human retinas in the imaging of OCT system. A transparent, non toxic and stable polymer, named PDMS, has been selected as the matrix material ${ }^{5}$ and its refractive index is $1.41 \pm 0.01$ in the near infrared region (800$1300 \mathrm{~nm})$, which is close to the index value of human retina $\left(n_{\text {retina }}=1.36\right)$. Due to the stability and size controllability of particles as well as relevant attenuation coefficient of particle concentration, ${ }^{16,17}$ Titanium dioxide $\left(\mathrm{TiO}_{2}\right)$ Nano powder has been selected as the scattered particle.

The concentration range of scattered particles was determined according to the relationship between attenuation coefficient and particle concentration using the mathematical model of SingleScattering. ${ }^{15,18,19}$ Firstly, the PDMS base material (SYLGARD 184, Dow Corning) was mixed homogeneously with $\mathrm{TiO}_{2}$ Nano powder $\left(\mathrm{TiO}_{2}\right.$, T104939, Aladdin). Seven different particle concentrations from $0.3 \%$ to $14 \%$ were used in this study. Secondly, the curing agent was added into the prepared $\mathrm{TiO}_{2}-\mathrm{PDMS}$ mixture with 1:10 mass ratio. Left in an oven with the $90^{\circ} \mathrm{C}$ environment for $3 \mathrm{~h}$, a piece of solid material can be obtained for subsequent operations. Finally, the singlescattering model ${ }^{15,18,19}$ was used to acquire the total attenuation coefficient of the materials. The relationship between the attenuation coefficient and the concentration of $\mathrm{TiO}_{2}$ particle material is shown in Fig. 1.

From the fit curve in Fig. 1, the saturation effect occurred with high concentration, therefore, in the process of preparing the materials, it became more and more difficult to keep the particle scattering homogeneously with the increase of the particle concentration. Through some experiments and measurements, it demonstrated that particle concentration should be lower than $4 \%$ to obtain an acceptable homogeneity. Meanwhile, the proper scattering signal intensity cannot be guaranteed without enough high particle concentration, therefore, the attenuation coefficient was required to exceed $4 \mathrm{~mm}^{-1}$. Considering the signal attenuation effects, three kinds of concentrations of $\mathrm{TiO}_{2}-$ PDMS materials, i.e., $2.8 \%, 1.8 \%$, and $1 \%$, were

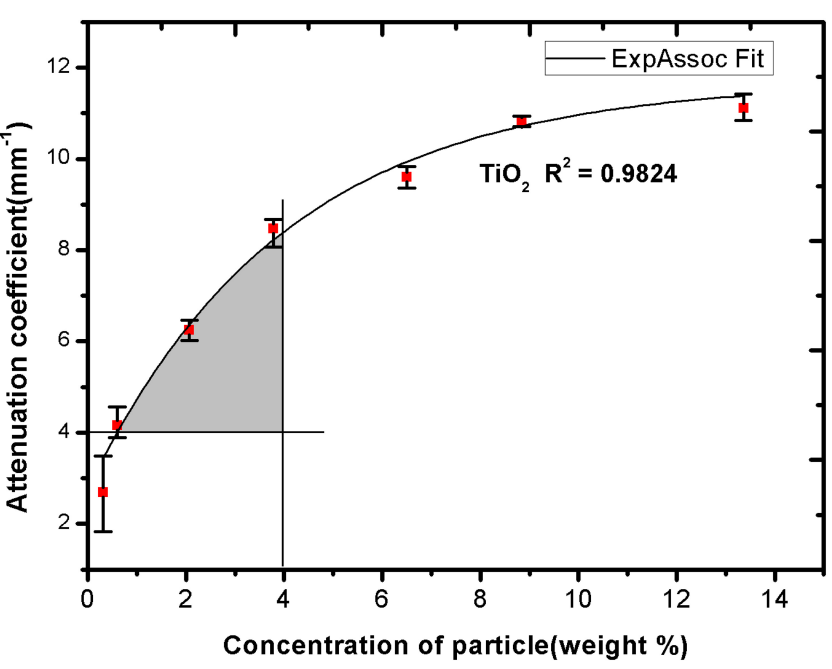

Fig. 1. The relationship between material attenuation coefficient and particle concentration.

selected to fabricate the multilayer structure with various layers corresponding to certain depths.

In order to prove the effects of using different particle concentrations, specific experiments were conducted and the measurement result was shown in Fig. 2. Firstly, the boundary of the arc surface

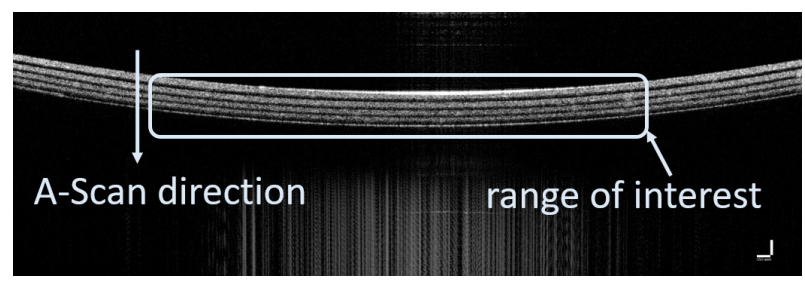

(a)

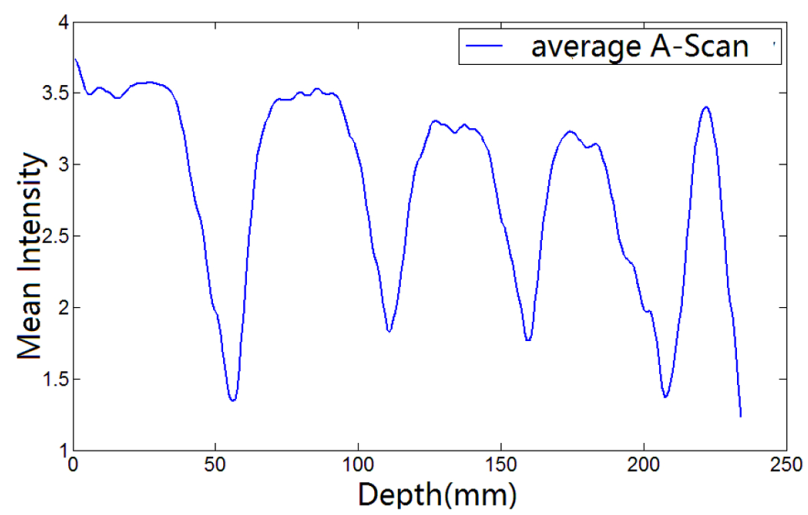

(b)

Fig. 2. Distribution of scattering intensity in depth. (a) B-scan of the multilayer structure by the OCT device. The range is 5800-1800 $\mu \mathrm{m}$, (b) Average A-Scan of the relationship between mean intensity and depth. 
was segmented through the MATLAB functions of boundary extraction, and then the radius could be calculated and the arc structure was straightened. Finally, the average A-Scan of specific region (Fig. 2(b)) can be calculated with the direction as Fig. 2(a). The mean intensity of A-Scan in Fig. 2(b) shows less signal attenuation in various layers with three kinds of particle concentrations.

\section{Design and Fabrication of Model Eyes}

\subsection{Architecture of model eyes}

After the crucial materials were selected through above-mentioned experiments, model eyes can be designed and fabricated to accomplish the function of detecting the axial resolution. As shown in Fig. 3, the shape of the model eye was designed similar to the natural one and the key optical parts including cornea, lens, vitreous body as well as retina were fabricated. ${ }^{2}$ As a transparent film, the cornea was made of glass and ultraprecise optical manufacturing method was applied to avoid dimension errors. The front cover and back cover were fabricated using $3 \mathrm{D}$ printing technology, and the vitreous body was composed of these two parts. Considering possible ocular dispersion and ametropic refraction, appropriate index-matching liquid can be injected into the vitreous body through the injection hole.

In order to ensure close fitting with the bottom of the vitreous body, the retina was simplified as the size of Fig. 3(d). Mimicking the real retina, the multilayer structure of the phantom was fabricated through spin coating with $\mathrm{TiO}_{2}-\mathrm{PDMS}$ and the detailed design and manufacturing procedure is described as follows.

\subsection{Design of the multilayer structure}

Analogous to the bars of the USAF 1951 resolution chart, the retina phantom was designed as curved multilayers (in Fig. 4) with progressive increase of the thicknesses for each layer pair (except for top and bottom layers), switching between brightness (with signals in OCT devices) and darkness (without signals in OCT devices). ${ }^{7}$ Among them, the bright layers have homogeneous particles, while no particles are in dark layers. The cross-section of the retina phantom with eight layers $(\mathrm{A}, 1,2,3,4,5,6, \mathrm{~B})$ is illustrated in Fig. 4. The thicknesses of Layers $\mathrm{A}$ and $\mathrm{B}$ are over $30 \mu \mathrm{m}$, aiming to protect the inner-layers (1-6) with standard thicknesses for detection. Meanwhile, in order to improve the efficiency of detection, three kinds of thicknesses were designed in the retina multilayer structure of a model eye. According to the survey of the axial resolutions of the current ophthalmic OCT devices, the thicknesses were designed as 9 to $30 \mu \mathrm{m}$.

\subsection{Fabrication of the multilayer structure}

In order to obtain the homogeneous distribution of particles and the clear boundaries between adjacent layers, the methods of ultrasonic cleaning and spin coating were utilized for the material preparation and fabrication of the multilayer structure. ${ }^{7}$ For the homogenization of the materials, $30 \mathrm{~min}$ vibration mixing and 10 to 20 min ultrasonic cleaning should be conducted before the curing agent was added into the prepared $\mathrm{TiO}_{2}-\mathrm{PDMS}$ mixture. As is well known, the film thicknesses are related to the spinning speed, time and material viscosity in the spin coating. In order to obtain thinner layers, $17 \%$

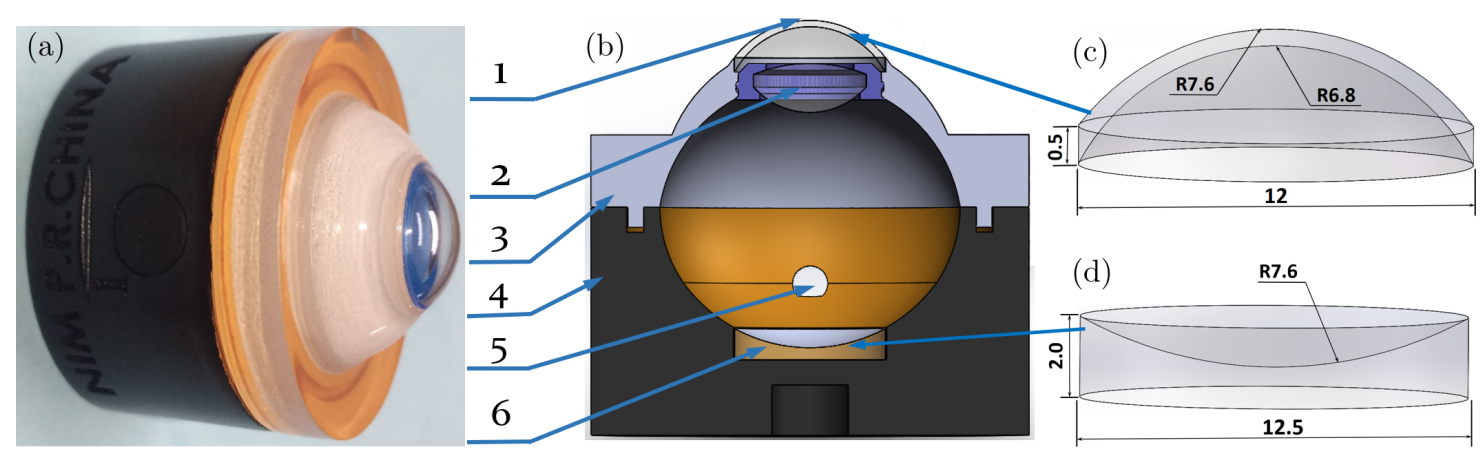

Fig. 3. The shape and architecture of the model eye. 1. Cornea, 2. Lens, 3. Front cover, 4. Back cover, 5. Injection hole, 6. Retina. (a) The actual model eye, (b) Cross-section structure of the model eye, (c) Enlarged drawing of 3D cornea and (d) Enlarged drawing of $3 \mathrm{D}$ retina. 


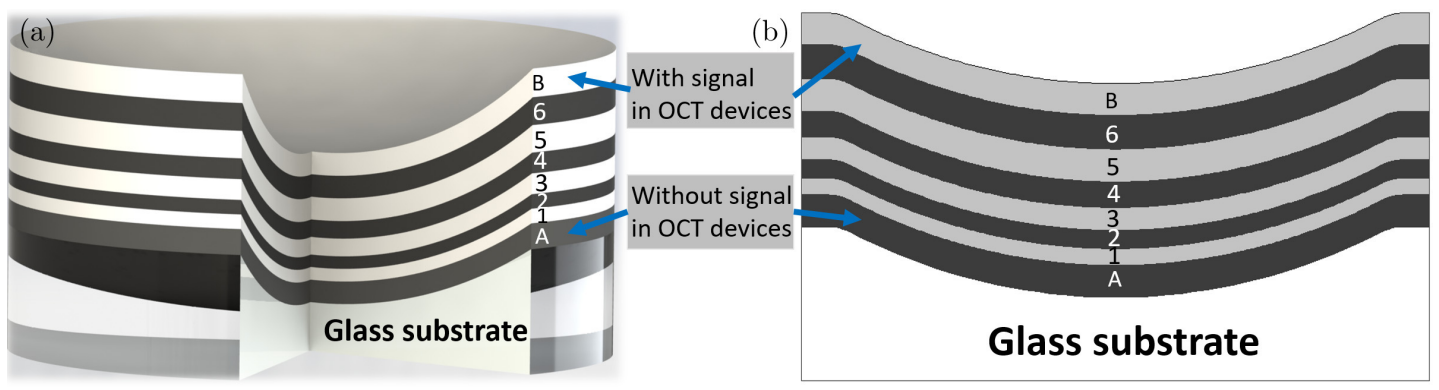

Fig. 4. View of the multilayer retina phantom. (a) The 3D model of layer structure with a $30^{\circ}$ section and (b) the cross-sectional view of the multilayer retina phantom.

(Weight) $n$-hexane was added in PDMS to reduce viscosity. Firstly, the glass substrate was stuck on a clean glass sheet which can be adsorbed on a spin coater. After each layer was spin coated, the sample was immediately baked on a hot plate with $180^{\circ} \mathrm{C}$ to avoid material shrinking and flowing down along the curved substrate surface and then the sample was cooled down to room temperature in $20 \mathrm{~min}$. Subsequently, the same procedure of the spin coating was repeated for other layers.

\section{The Measurement Result}

\subsection{Thickness calibration}

For the calibration, a thickness monitoring system (Si Wafer Thickness Monitoring System SF-3, Otsuka Electronics, Japan) was applied after the model eyes were accomplished. It should be noted that the employed monitoring system could not calibrate the thicknesses of each layer of the whole multilayer structure at one time. However, the total thickness of the multilayer structure can be measured after each spin coating process and then the thickness of each layer can be calculated through subtraction of the total value and the previous one. The results of the measurements and calculations are shown in Table 1 with three model eyes (M1, M2, and M3). In order to avoid the measurement error of the thickness monitoring system, each calibration result was calculated through four repeated measurements after one spin coating process. The calibration results and design values of each layer show that the fabrication deviation can be controlled within $5 \%$.

\subsection{Measurements with the OCT systems}

Analogous to USAF 1951 resolution chart, the axial resolution of an OCT device is defined as the value of the minimal thickness, of which the mean intensity of the bright (peak) and dark (trough) layer can be distinguished in the A-Scan images of the OCT device. In this study, the fabricated three model eyes (M1, M2 and M3) were used to evaluate the axial resolution of three OCT devices, including two following research-grade OCT system: a spectral domain OCT device (THORLABS, USA) and a selfestablished swept source OCT device, and a clinical ophthalmic OCT device (OCT 3D-1000, TOPCON, Japan), and the results of the cross-sectional images (B-Scan) are shown in Fig. 5. The mean intensity of A-Scan of cross-sectional images (B-Scan) is also shown in Fig. 5 through the MATLAB functions.

According to Fig. 5, the axial resolution of the devices can be quickly obtained through the A-Scan

Table 1. Comparison of the design value and calibration result for each layer thickness.

\begin{tabular}{|c|c|c|c|c|c|c|c|c|c|}
\hline \multicolumn{2}{|c|}{ Layer thickness $(\mu \mathrm{m})$} & \multirow{2}{*}{$\begin{array}{c}\mathrm{a} \\
>30 \\
34.07\end{array}$} & \multirow{2}{*}{$\begin{array}{c}1 \\
20 \\
20.35\end{array}$} & \multirow{2}{*}{$\begin{array}{c}2 \\
20 \\
19.61\end{array}$} & \multirow{2}{*}{$\begin{array}{c}3 \\
25 \\
24.45\end{array}$} & \multirow{2}{*}{$\begin{array}{c}4 \\
25 \\
24.76\end{array}$} & \multirow{2}{*}{$\begin{array}{c}5 \\
30 \\
29.96\end{array}$} & \multirow{2}{*}{$\begin{array}{c}6 \\
30 \\
29.70\end{array}$} & \multirow{2}{*}{$\begin{array}{c}\mathrm{b} \\
>30 \\
35.18\end{array}$} \\
\hline M1 & $\begin{array}{c}\text { Design value } \\
\text { Calibration result }\end{array}$ & & & & & & & & \\
\hline M2 & $\begin{array}{c}\text { Design value } \\
\text { Calibration result }\end{array}$ & $\begin{array}{c}>30 \\
33.48\end{array}$ & $\begin{array}{c}12 \\
12.37\end{array}$ & $\begin{array}{c}12 \\
12.55\end{array}$ & $\begin{array}{c}15 \\
15.37\end{array}$ & $\begin{array}{c}15 \\
14.47\end{array}$ & $\begin{array}{c}18 \\
17.32\end{array}$ & $\begin{array}{c}18 \\
18.46\end{array}$ & $\begin{array}{r}>30 \\
35.18\end{array}$ \\
\hline M3 & $\begin{array}{c}\text { Design value } \\
\text { Calibration result }\end{array}$ & $\begin{array}{c}>30 \\
34.35\end{array}$ & $\begin{array}{c}9.5 \\
9.23\end{array}$ & $\begin{array}{c}9.5 \\
9.53\end{array}$ & $\begin{array}{c}10.5 \\
10.84\end{array}$ & $\begin{array}{c}10.5 \\
10.58\end{array}$ & $\begin{array}{c}>30 \\
36.12\end{array}$ & - & - \\
\hline
\end{tabular}


SD

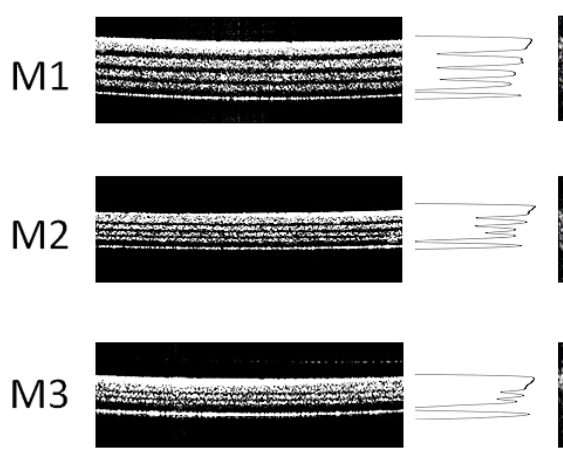

SSD
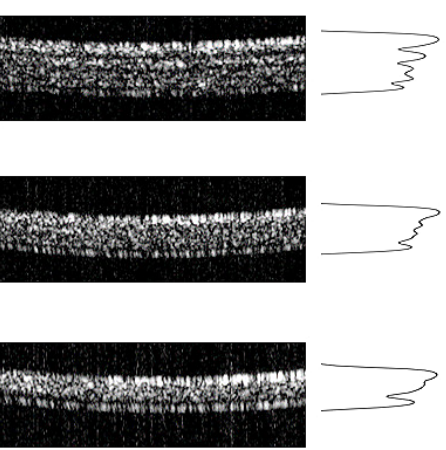

$C D$
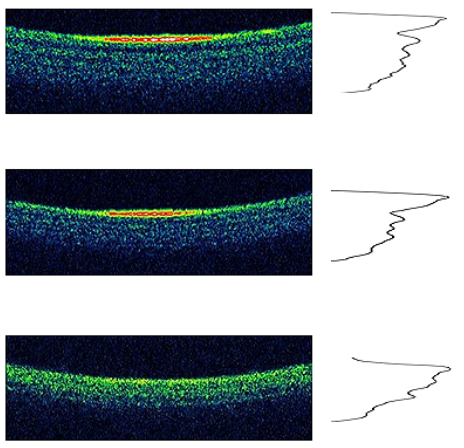

Fig. 5. Diagram of measuring axial resolution. M1, M2 and M3 are the model eyes with various layer thicknesses. SD: spectral domain OCT device, SSD: swept source OCT device, CD: clinical OCT device.

and B-Scan images. for example, all the peak and the trough of bright and dark layer pairs of the total three model eyes can be distinguished for the SD device, it means its axial resolution is less than $9.5 \mu \mathrm{m}$. Meanwhile, since the minimal peak and trough signal which can be distinguished by the SSD and CD device are respectively $15 \mu \mathrm{m}$ (Layers 3 and 4 in M2 for the SSD) and $10.5 \mu \mathrm{m}$ (Layers 3 and 4 in M3 for the CD), their axial resolutions are, respectively, $15 \mu \mathrm{m}$ and $10.5 \mu \mathrm{m}$. Considering the device development is still in progress, the selfestablished SSD can be further improved in the parameter of axial resolution compared with the SD. For the clinical OCT instrument, it could be concluded that the device performance has deteriorated badly compared to its original axial resolution of $6.5 \mu \mathrm{m}$, and it needs to be sent for equipment service.

\section{Discussion and Conclusion}

In this study, novel model eyes with curved multilayer structure for the axial resolution evaluation of an ophthalmic OCT device were designed and manufactured. As reported in recent literature, PSF has the problem of the complicated post-process, while phantoms have the disadvantage of difficult adjustment in evaluation process, hence, they were not suitable for clinical applications. Compared with these methods, our model eyes with the multilayer films can be used to rapidly evaluate clinical OCT devices without complicated process. Though Agrawal et al. proposed the detection method using multilayer structure, ${ }^{7}$ it still has some disadvantages including inaccuracy with signal attenuation, and low efficiency due to the same thickness of each layer in the phantom. In our model eyes, some novel methods have been proposed: (1) Similar to natural human eyes, the OCT model eyes consist of optical tissue elements including cornea, lens, vitreous body and curved retina, ensuring the integrity of the detection phantom, which is important for clinical applications; (2) the multilayer structure in the retina is fabricated using TiO2PDMS. Through the experiments, the various layers with different scattering coefficient are designed, which can reduce the effect of signal attenuation and prevent the evaluation inaccuracy; (3) Three kinds of thicknesses are designed in the multilayer structure for one model eye, therefore three levels of resolution can be detected in single measurement and the detection efficiency can be greatly improved. In addition, in order to guarantee the traceability and accuracy of the key parameters of the standard model eyes, the thicknesses of the multilayer structure of artificial retina are verified with the thickness monitoring system, and the fabrication deviations are controlled within $5 \%$.

The model eyes presented in this study could be spread to assist the evaluation of the axial resolution of ophthalmic OCT equipment. It will be available for OCT researchers and clinicians to use for regular quality control. It also should be admitted that thinner layers are not currently available due to viscosity between adjacent layers. Novel fabrication methods as well as different materials are investigated to acquire film with thickness below OCT theoretical resolution limit. For the future work, the multilayer uniformity and process stability still needs to improve. Meanwhile, the lower 
limit of the layer thickness should be extended to sub-micron level, thereby be able to assess the emerging new OCT devices with super high resolution.

\section{Conflict of Interest}

No conflicts of interest, financial or otherwise, are declared by the authors.

\section{Acknowledgments}

The study was supported by the National Key Research and Development Program of China (2016YFF0201005). We would like to thank Zhixiong $\mathrm{Hu}$ and Xiaojun Chen for constructive review of the paper. In addition, we would like to thank Zengqian Ding and Wen Qiao from the Soochow University, for their contribution to this work.

\section{References}

1. D. Huang et al., "Optical Coherence Tomography," Science (New York, N.Y.) 254(5035), 1178-1181 (1991).

2. "ISO 16971-2015-04-15," (2015).

3. Z. Hu et al., "A physical model eye with $3 \mathrm{D}$ resolution test targets for optical coherence tomography," Opt-Electron. Eng. 41(12), 28-32 and 38 (2014). doi:10.1117/12.2067974.

4. A. Agrawal et al., "Three-dimensional characterization of optical coherence tomography point spread functions with a nanoparticle-embedded phantom," Opt. lett. 35(13), 2269 (2010). doi:10.1364/OL.35.002269.

5. T. Wen et al., "A standard test method based on point spread function for three-dimensional imaging system," Proc. SPIE, pp. 96842F-96842F-9 (2016). doi: $10.1117 / 12.2243260$.

6. A. Agrawal et al., "Characterizing the point spread function of retinal OCT devices with a model eyebased phantom," Biomed. Opt. Express 3(5), 1116 (2012). doi:10.1364/BOE.3.001116.

7. A. Agrawal et al., "Multilayer thin-film phantoms for axial contrast transfer function measurement in optical coherence tomography," Biomed. Opt. Express 4(7), 1166 (2013). doi:10.1364/BOE.4.001166.
8. T. S. Rowe, R. J. Zawadzki, "New developments in eye models with retina tissue phantoms for ophthalmic optical coherence tomography," Proc. SPIE, 8229(1) (2012). doi:10.1117/12.905499.

9. T. S. Rowe, R. J. Zawadzki, "Development of a corneal tissue phantom for anterior chamber optical coherence tomography (AC-OCT)," SPIE BiOS. International Society for Optics and Photonics, pp. 85830I-85830I-9, 148-156 (2013). doi:10.1117/12.2005744.

10. J. Baxi et al., "Retina-simulating phantom for optical coherence tomography," J. Biomed. Opt. 19(2), 021106-021106 (2013). doi:10.1117/1.JBO.19.2.021106.

11. R. Y. Gu et al., "Variable-sized bar targets for characterizing three-dimensional resolution in OCT," Biomed. Opt. Express 3(9), 2317 (2012). doi:10.1364/BOE.3.002317.

12. A. Curatolo et al., "Structured three-dimensional optical phantom for optical coherence tomography," Opt. Exp. 19(20), 19480 (2011). doi:10.1364/ OE.19.019480.

13. P. H. Tomlins et al., "Femtosecond laser microinscription of optical coherence tomography resolution test artifacts," Biomed. Opt. Exp. 2(5), 1319 (2011). doi:10.1364/BOE.2.001319.

14. Z. $\mathrm{Hu}$ et al., "Test target for characterizing $3 \mathrm{D}$ resolution of optical coherence tomography," Proc. SPIE, pp. 92971S-92971S-7 (2014). doi:10.1117/ 12.2073062 .

15. G. Lamouche et al., "Review of tissue simulating phantoms with controllable optical, mechanical and structural properties for use in optical coherence tomography," Biomed. Opt. Express 3(6), 18 (2012). doi:10.1364/BOE.3.001381.

16. D. M. de Bruin et al., "Optical phantoms of varying geometry based on thin building blocks with controlled optical properties," J. Biomed. Opt. 15(2), 025001-025001-025010 (2010). doi:10.1117/1.3369003.

17. B. W. Pogue, M. S. Patterson, "Review of tissue simulating phantoms for optical spectroscopy, imaging and dosimetry," J. Biomed. Opt. 11(4), 041102-041102-041116 (2006). doi:10.1117/1.2335429.

18. C. E. Bisaillon, M. L. Dufour, G. Lamouche, "Artery phantoms for intravascular optical coherence tomography: Healthy arteries," Biomed. Opt. Express 2(9), 2599-2613 (2011). doi:10.1364/BOE.2.002599.

19. M. Hammer et al., "Optical properties of ocular fundus tissues determined by optical coherence tomography," Opt. commun. 186(1-3), 149 (2000). doi:10.1016/S0030-4018(00)01054-3. 